\title{
Pre-Triangulations and Liftable Complexes
}

\author{
Oswin Aichholzer • Franz Aurenhammer • \\ Thomas Hackl
}

Received: 2 December 2005 / Revised: 7 August 2007 /

Published online: 13 September 2007

(C) Springer Science+Business Media, LLC 2007

\begin{abstract}
We introduce the concept of pre-triangulations, a relaxation of triangulations that goes beyond the frequently used concept of pseudo-triangulations. Pretriangulations turn out to be more natural than pseudo-triangulations in certain cases. We show that pre-triangulations arise in three different contexts: In the characterization of polygonal complexes that are liftable to three-space in a strong sense, in flip sequences for general polygonal complexes, and as graphs of maximal locally convex functions.
\end{abstract}

Keywords Pre-triangulations · Pseudo-triangulations · Liftable complexes

\section{Introduction}

Polygonal complexes in the plane have been objects of interest in combinatorial geometry from various points of view. With the advent of computational geometry, it soon became apparent that combinatorial and geometric properties of certain polygonal complexes prove useful for structuring geometric data and designing efficient algorithms. Classical examples are line arrangements that arise as duals of finite point sets [9], Voronoi diagrams that capture proximity information among geomet-

Research supported by the FWF Joint Research Project 'Industrial Geometry' S9205-N12.

O. Aichholzer · T. Hackl

Institute for Software Technology, Graz University of Technology, Graz, Austria

O. Aichholzer

e-mail: oaich@ist.tugraz.at

T. Hackl

e-mail: thackl@ist.tugraz.at

F. Aurenhammer $(\varangle)$

Institute for Theoretical Computer Science, Graz University of Technology, Graz, Austria

e-mail: auren@igi.tugraz.at 
ric objects $[2,11]$, and triangulations that connect or partition geometric objects in predefined domains $[6,11]$.

Whereas generalizations of line arrangements and Voronoi diagrams meanwhile have been studied extensively, the discovery of a structure that generalizes triangulations but still retains their basic properties (e.g., planarity, simple face shape, and flippability) happened more recently. In a so-called pseudo-triangulation, faces bounded by three reflex chains, rather than by three line segments, are allowed. Pseudo-triangulations enjoy a variety of combinatorial and geometric properties, and lead to efficient data structures and algorithms in several areas. See, for example, $[1,19,20,24]$ and references therein, respectively.

The aim of this paper is to generalize triangulations in a natural way beyond pseudo-triangulations. A pseudo-triangle is a simply connected polygonal region where exactly three vertices have no reflex angle. Dropping simplicity, we arrive at a concept we will call a pre-triangle, and following suit, a pre-triangulation of a given domain. We show that pre-triangulations arise in three different contexts: In the characterization of complexes that are liftable to three-space in a strong sense, in flip sequences for general polygonal complexes, and as graphs of maximal locally convex functions. Below we give some background on these topics and briefly outline our results.

\subsection{Liftable Complexes}

The issue of characterizing complexes that can be 'lifted' to space has been frequently investigated in the mathematical literature. A classical theorem of Steinitz [23] implies that, for every complex in the plane whose edge graph is three-connected, there exists a convex 3-polyhedron with isomorphic boundary. The Maxwell-Cremona theorem, see e.g. [8], characterizes polygonal complexes whose edges are exactly the vertical projections of the edges of a polyhedral surface. Complexes with this property are sometimes called projective or liftable complexes. When the projection surface is required to be convex, the well-studied class of regular complexes is obtained. Several criteria for characterizing regular complexes (in general dimensions) exist; see e.g. [3] for a short bibliography. Deciding liftability is also a question of practical importance, for example, in scene analysis.

In the present paper, we are interested in a concept of liftability which is robust with respect to variations in lifting heights, and, at the same time, outrules complexes that are (not) liftable only because of geometric artifacts. We introduce the notion of combinatorial projectivity, which informally means that after a random $\varepsilon$-perturbation of the complex, the space of exact lifts has the maximum possible dimension with probability one. Interestingly, this dimension is related to the number of those vertices in the complex where the angles at all their incident faces are convex. This number we will call the degree of the complex. Every polygonal complex has an exact lifting dimension at most its degree, and there are complexes achieving this bound. Combinatorial projectivity is, thus, a property of the graph of edges of the complex and of the order type ${ }^{1}$ of its vertices, rather than of the geometry of the vertices and their surface heights.

\footnotetext{
${ }^{1}$ The order type [12] of a finite set $S$ of points in the plane assigns to each ordered triple $\{p, q, r\} \subset S$ its orientation, either clockwise or counterclockwise. 
Fig. 1 Schlegel diagram with perturbed vertex

Fig. 2 Pseudo-triangulation with flat surface edge
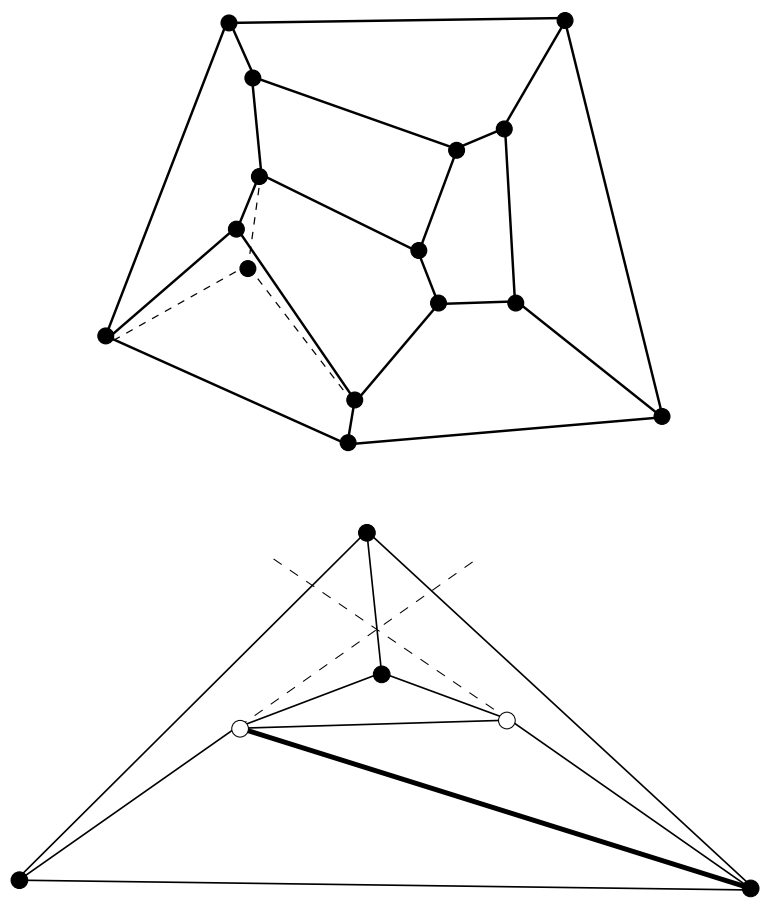

To see some examples, triangulations are projective complexes that are also combinatorial projective (though they are not necessarily regular). Schlegel diagrams [13], and thus Voronoi diagrams and power diagrams [3], are regular and therefore projective. However, these complexes are not combinatorial projective unless they are triangulations: A movement of vertices of degree three will typically destroy their projectivity, and thus the existence of an exact lifting surface; see Fig. 1. Also, non-triangular faces will split for most choices of heights for surface vertices. Especially, complexes consisting of a single polygonal face are not combinatorial projective, in general. Their space of exact lifts is of dimension only three, even for convex $n$-gons (where only convex angles occur at vertices, such that the above-mentioned bound on the lifting dimension is $n$ ). Clearly, such complexes are projective, but an exact lift exists only for vertex heights with special geometry.

On the other hand, the complex shown in Fig. 2 (taken from [1]) is not projective, because the edge drawn in bold flattens out in all possible projection surfaces: The two triangles sharing this edge always lie in the same plane, which concurs with the two adjacent planes at the point where the dotted lines cross. But $\varepsilon$-perturbing almost surely restores projectivity in this case. Moreover, this complex is combinatorial projective, as an exact lifting surface will exist for almost all choices of heights for the vertices where all incident angles are convex (emphasized in black).

In Sects. 3 through 6 we derive a criterion that completely characterizes when a given polygonal complex is combinatorial projective. This characterization hinges on the concept of pre-triangulations, introduced in Sect. 3. More specifically, a complex is combinatorial projective if and only if it is identical to its $M$-skeleton, a certain pretriangulation defined in Sect. 4. In that section we also show that the surface theorem 


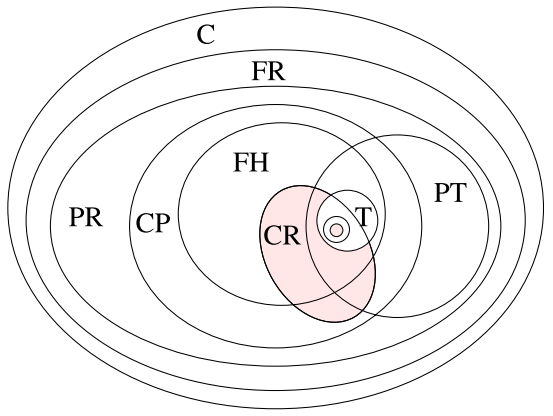

$$
\begin{array}{cll}
\text { C } & \ldots & \text { polygonal complexes } \\
\text { FR } & \ldots & \text { face-reducible complexes } \\
\text { PR } & \ldots & \text { pre-triangulations } \\
\text { CP } & \ldots & \text { combinatorial projective complexes } \\
\text { FH } & \ldots & \text { face-honest complexes } \\
\text { CR } & \ldots & \text { combinatorial regular complexes } \\
\text { PT } & \ldots & \text { pseudo-triangulations } \\
\text { T } & \ldots & \text { triangulations } \\
\bigcirc & \ldots & \text { Delaunay triangulations constrained by } R \\
\text { (shaded) } & \ldots \text { area for Delaunay minimum complex of } R
\end{array}
$$

Fig. 3 Hierarchy of polygonal complexes in a given region $R$

for pseudo-triangulations in [1] holds in a more general setting. Loosely speaking, this theorem asserts that three (non-trivial) vertex heights per face can be chosen in any given pseudo-triangulation such that each face lifts to planarity. We characterize the class of complexes where the surface theorem applies. This leads to the class of so-called face-reducible complexes which contains the pre-triangulations as a proper subclass.

In Sect. 7 we turn to convex projection surfaces and define combinatorial regularity, a property stronger than combinatorial projectivity. We introduce the class of facehonest complexes, which are basically those where each single face can be lifted to a different plane. Both face-honest complexes and combinatorial regular complexes are certain pre-triangulations. Combinatorial regular complexes in simply connected regions are shown to be face-honest pseudo-triangulations. The various considered complex classes and their containment relations are illustrated in Fig. 3. The picture is nonredundant in the sense that subclasses are proper and class overlaps are nonempty.

\subsection{Flips and Convexity}

One of the most basic properties of convex sets is their facial structure $[7,13]$. In this sense, every convex and piecewise linear function generates a cell complex in its domain of definition. For example, the maximal convex function that does not exceed certain prescribed values on a finite set of points in the plane leads to a complex whose faces are polygonal. This insight dates back to the classical observation that Delaunay triangulations are projected lower convex hulls; see e.g. [2, 11]. A similar relation exists in a more general setting [1]: Maximal locally convex functions generate constrained regular pseudo-triangulations if the domain of definition is a nonconvex polygon. We extend the situation to more general domains. In Sect. 8 we prove that graphs of locally convex functions on arbitrary polygonal domains (with possible holes) generate combinatorial regular complexes, and thus pre-triangulations, in the generic case. This result turns out useful in the design of a new and powerful flipping operation for polygonal complexes, whose properties are studied in Sects. 9 to 12.

So-called flips are a frequently used means to modify polygonal complexes. Various types of flip operations have been considered in the literature, mainly for triangulations and pseudo-triangulations. To give a few relevant citations, we refer to 
Fig. 4 Exchanging flips
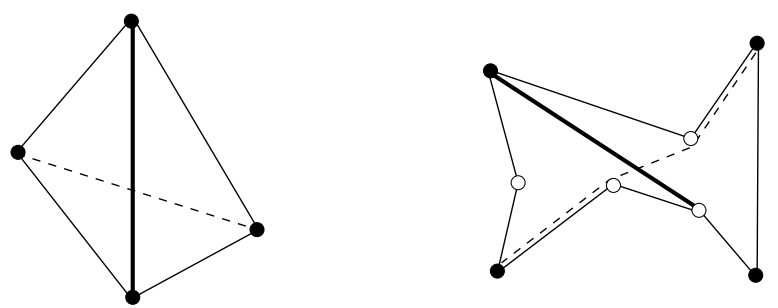

Fig. 5 Removing flips

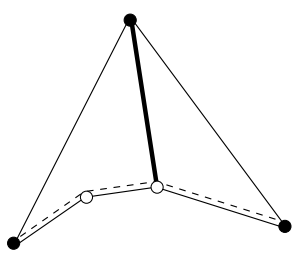

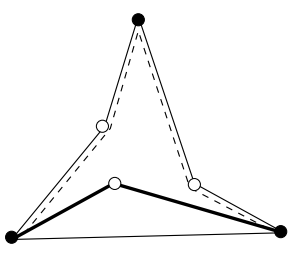

$[10,15,16]$ and $[1,19,20,24]$, respectively. All these flips can be defined via geodesic lines in the domain where the flip takes place.

Figure 4 shows the standard Lawson flip for triangulations (left) and the exchanging flip for pseudo-triangulations (right). An edge-removing flip and a vertexremoving flip are illustrated in Fig. 5 left and right, respectively. In both figures, the edges to be flipped are drawn in bold, and the geodesic lines that define the edges created in the flip (if any) are shown dashed. The class of pseudo-triangulations is closed under flips of these four types.

Extending a different approach, taken in [1] and based on locally convex functions rather than on geodesic lines, we derive a general flip operation in Sects. 9 and 10. This operation works for the entire class of face-reducible complexes and covers all the classical flip types. The smallest class (from the classes listed in Fig. 3) that is closed under this operation are the pre-triangulations.

In Sect. 11 we introduce the Delaunay minimum complex, which is a variant of the well-known Delaunay triangulation $[2,11]$ for general polygonal domains. This (unique) structure is the complex of smallest combinatorial size that still retains the desired 'Delaunay properties': an analog of local Delaunayhood, and reachability by improving flips. The Delaunay minimum complex is strongly related to the concept of constrained regular pseudo-triangulations introduced in [1], and generalizes the concept of pointed Delaunay pseudo-triangulation in [22]. We prove that any given face-reducible complex in a polygonal region $R$ can be flipped to the Delaunay minimum complex of $R$ by means of improving flips. This connectivity result is a generalization of the optimality theorem in [1]. Section 12 shows that, within simply connected regions, every triangulation can be flipped to a predefined constrained regular pseudo-triangulation, in a way such that all intermediate complexes are facehonest pseudo-triangulations (except immediately before a vertex is removed). Flip sequences that retain face-honesty are desirable because they change the complex at hands in a local way. 


\section{Polygonal Complexes}

This section provides the definitions and notions we will use to work on polygonal complexes.

Let $R$ be a bounded subset of the plane. We call $R$ a polygonal region if the boundary of $R$ is piecewise linear and coincides with the boundary of the interior of $R$. Neither connectedness nor simple connectedness of a polygonal region $R$ is required. The boundary components of $R$ are called edges and vertices of $R$. A corner of $R$ is a vertex of $R$ with no internal angle larger than $\pi$. All other vertices of $R$ are termed noncorners of $R$. See Fig. 6. The depicted region consists of three connected components, two being simply connected. Corners and noncorners are distinguished as black dots and white dots. Note that more than one internal angle may arise at a single vertex. For corners, all these angles have to be convex.

A polygon is a polygonal region that is homeomorphic to a disk. Observe that the convex hull of a polygonal region $R$ is a convex polygon whose vertices are corners of $R$. This implies that every polygonal region has at least 3 corners.

A polygonal partition, $\mathcal{C}$, of $R$ is a partition of $R$ into (finitely many) polygonal regions. Such a region $f$ is called a face of $\mathcal{C}$ if the interior of $f$ is connected. Faces need not be simply connected; they may contain holes and thus are not polygons, in general. The edges and vertices of $\mathcal{C}$ are the edges and vertices of its faces. An edge (vertex) of $\mathcal{C}$ is called internal (to $R$ ) if it does not lie on the boundary of $R$. $\mathcal{C}$ is termed a polygonal complex in $R$ if each internal edge is an edge of two different faces.

We will restrict attention to polygonal complexes in this paper. The vertices of any polygonal complex $\mathcal{C}$ are assumed to be in general position ${ }^{2}$ in the plane. As the vertices of the underlying polygonal region $R$ arise as vertices of $\mathcal{C}$, the vertices of $R$ are required to be in general position as well.

Consider an arbitrary subset $B$ of faces of a polygonal complex $\mathcal{C}$. Let $v$ be a vertex of some face in $B$. Adopting notation from [1], vertex $v$ is called complete in $B$ if $v$ is a corner of each face in $B$ that is incident to $v$. Otherwise, $v$ is called incomplete in $B$. Note that, if $v$ is incomplete in $B$, then there is a unique face in $B$ where the

Fig. 6 General polygonal region

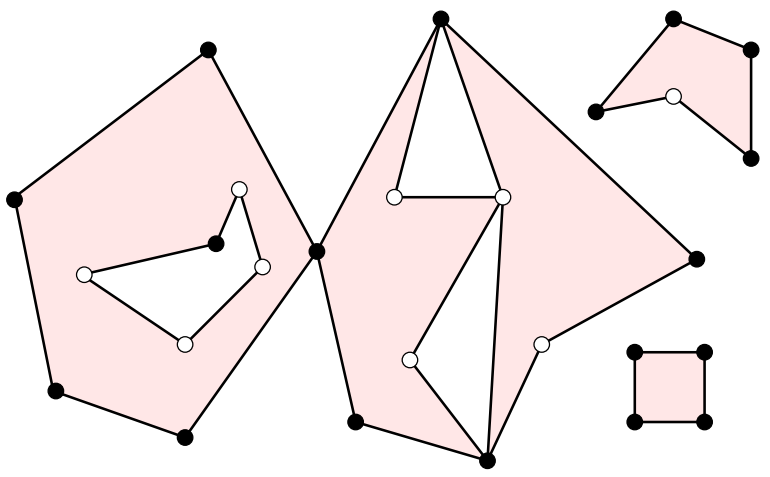

\footnotetext{
${ }^{2}$ A set $S$ of points in the plane is in general position if no 3 points of $S$ are collinear. 
Fig. 7 A polygonal complex

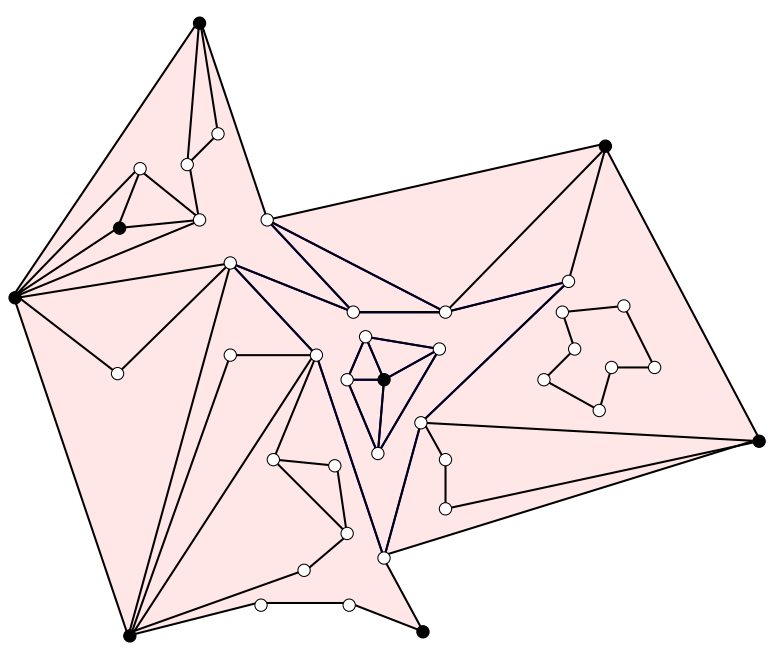

internal angle at $v$ is larger than $\pi$. For vertices of $\mathcal{C}$ which are not incident to some face in $B$, the completeness status with respect to $B$ is left undefined. Distinguishing complete and incomplete vertices will be crucial in deciding liftability of a complex.

For notational convenience, we will mostly write a polygonal complex $\mathcal{C}$ as the set of its faces. If a vertex $v$ is complete in $\mathcal{C}$, then there is no subset $B$ of faces of $\mathcal{C}$ where $v$ is incomplete. Equivalently, if $v$ is incomplete in $B \subset \mathcal{C}$, then $v$ is also incomplete in $\mathcal{C}$. We define the degree of $B$ as the number of vertices of $\mathcal{C}$ that are complete in $B$. The notion of degree of a face set is central for the developments in the present paper. Notice that the union of the faces in $B$ is a polygonal region, and that each corner of this region has to be complete in $B$. This implies that the degree of $B$, and in particular the degree of $\mathcal{C}$, is at least 3 .

Figure 7 shows a polygonal complex of degree 8 . Complete and incomplete vertices are drawn as black dots and white dots, respectively. We will keep this convention (which is also compatible with Figs. 1, 2, and 6) throughout this paper. Observe that the (in)completeness of a vertex is a property based on the underlying complex, whereas the (non)corner property of a vertex is based on the respective polygonal region. Note finally that adjacent faces of a complex may touch at many edges, but each internal edge has to belong to exactly two faces.

\section{Pre-Triangulations}

This section introduces the concept of pre-triangulation. We start by recalling the (related) definition of a pseudo-triangulation [1, 19, 20, 24]. We then define minimum complexes in polygonal regions, and finally give a characterization of face sets of degree 3. Such face sets will turn out important in most of our subsequent investigations.

A pseudo-triangle is a polygon with exactly 3 corners. A pseudo-triangulation is a polygonal complex all whose faces are pseudo-triangles. 


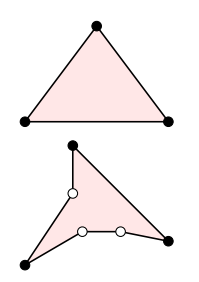

(a)

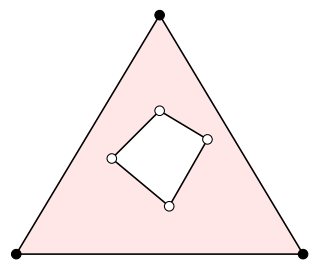

(b)

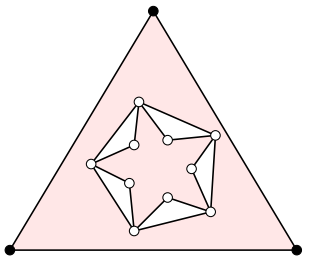

(c)

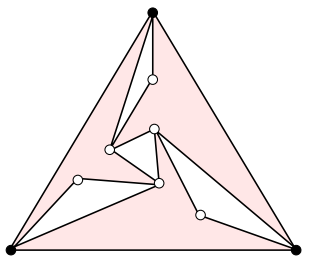

(d)

Fig. 8 Five valid pre-triangles

Fig. 9 M-skeleton of the face-reducible complex in Fig. 7

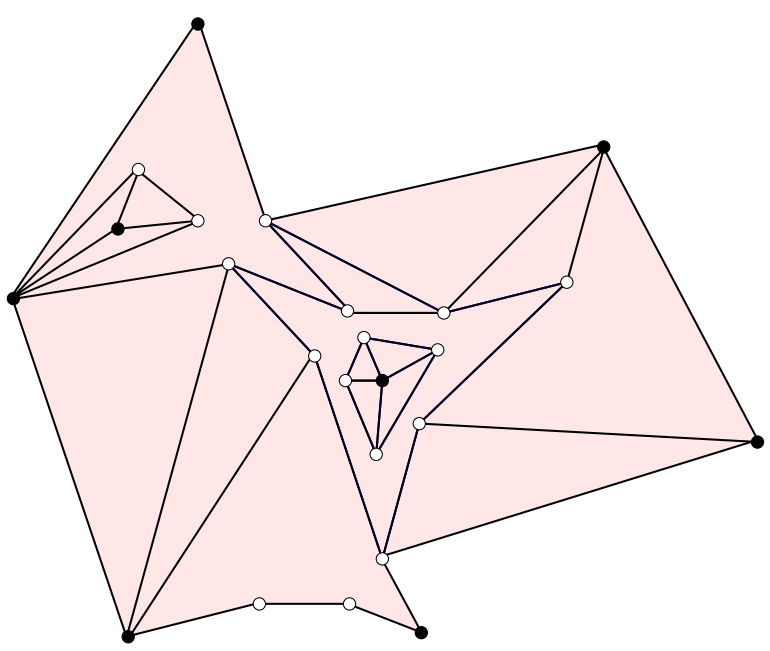

We define a pre-triangle as an arbitrary polygonal region with exactly 3 corners. Clearly, any pseudo-triangle is a pre-triangle, but the latter may contain convex holes, because no vertex of such a hole is an additional corner. In fact, the interior of a pretriangle may be disconnected, as it may consist of many edge-disjoint faces. See Fig. 8. Part (a) illustrates two pseudo-triangles, whereas parts (b), (c), and (d) show pre-triangles which are not pseudo-triangles. The pre-triangle in (c) consists of two faces, and the pre-triangle in (d) consists of three faces.

A polygonal complex $\mathcal{C}$ is called a pre-triangulation if $\mathcal{C}$ can be partitioned into subsets $B_{1}, \ldots, B_{t}$ of faces such that (1) the union of the faces in each $B_{i}$ is a pre-triangle, and (2) the faces of each $B_{i}$ are pairwise edge-disjoint. Clearly, every pseudo-triangulation is also a pre-triangulation. However, a pre-triangulation may contain faces with holes (Fig. 9) and even faces with a large number of corners (Fig. 8(c)).

Let $\mathcal{C}$ be some polygonal complex. Recall that each corner of the underlying polygonal region $R$ is complete in $\mathcal{C}$. This leads us to term $\mathcal{C}$ a minimum polygonal complex if the corners of $R$ are the only vertices that are complete in $\mathcal{C}$. In other words, the degree of $\mathcal{C}$ equals the number of corners of $R$, the minimum that can be achieved. Observe that, in a minimum complex, every internal vertex (if any) is reflex in one of 
its incident faces. To give examples, the complex in Fig. 13 is minimum whereas the complexes in Figs. 7 and 9 are not.

For pseudo-triangulations, the definition above is consistent with the original definition that uses vertex pointedness: In a minimum pseudo-triangulation [24] each vertex is pointed, that is, its incident edges span a convex angle. Observe that a polygonal complex $\mathcal{C}$ in $R$ is minimum if and only if $\mathcal{C}$ can be 'filled up' to a minimum pseudo-triangulation in $R$ by adding edges between vertices of $\mathcal{C}$. In particular, the number of edges of a minimum complex in $R$ is not determined by its vertex set. The natural counterpart to minimum complexes are complexes where all vertices are complete. Triangulations and Schlegel diagrams (Fig. 1) are notable examples.

The following lemma characterizes face sets of degree 3 in a polygonal complex $\mathcal{C}$. Here and in later sections we denote with $U(B)$ the union of the faces in a subset $B \subset \mathcal{C}$.

Lemma 1 Let $B$ be any subset of faces of $\mathcal{C}$. Then $B$ is of degree 3 if and only if $U(B)$ is a pre-triangle and $B$ forms a minimum polygonal complex in $U(B)$.

Proof Assume that $B$ is of degree 3. As $U(B)$ has at least 3 corners, and each such corner is complete in $B$, we conclude that $U(B)$ has exactly 3 corners. That is, $U(B)$ is a pre-triangle, and the polygonal complex formed by $B$ in $U(B)$ is minimum.

Conversely, assume that $U(B)$ is a pre-triangle, and that the polygonal complex formed by $B$ in $U(B)$ is minimum. Then the degree of this complex equals the number of corners of $U(B)$, which is 3 because $U(B)$ is a pre-triangle. That is, $B$ is of degree 3 .

\section{The M-Skeleton}

Utilizing pre-triangulations, we now define a substructure for polygonal complexes, the so-called M-skeleton, which is the key to combinatorial projectivity. Intuitively speaking, the M-skeleton delimitates maximal face sets of the complex that lift to planarity in a robust sense. We introduce the class of face-reducible complexes and demonstrate that the surface theorem for pseudo-triangulations in [1] can be extended to this more general class. Face-reducible complexes are also the largest class where this is possible, as Sect. 6 shows.

A polygonal complex is termed face-reducible if and only if each of its faces is contained in some subset of faces of degree 3. For example, every pre-triangulation (and, in particular, every (pseudo-)triangulation) is a face-reducible complex. Note that such complexes may contain faces of any shape. Figure 7 gives an illustration. The reader is encouraged to check that this complex is face-reducible (using Fig. 9 as an aid).

Let $\mathcal{C}$ be a face-reducible complex for the rest of this section. For a face $f \in \mathcal{C}$, we will denote with $M_{f}$ a maximal subset of faces of $\mathcal{C}$ that contains $f$ and that is of degree 3. Recall from Lemma 1 that $U\left(M_{f}\right)$ is a pre-triangle. We state and prove two basic properties of such maximal face sets. 
Lemma 2 Let $f, g \in \mathcal{C}$. If $M_{f} \cap M_{g} \neq \emptyset$ then $M_{f}=M_{g}$.

Proof Let $M_{f} \cap M_{g} \neq \emptyset$. We claim that $M_{f} \cup M_{g}$ is of degree 3. This implies $M_{f}=$ $M_{g}$ by the maximality of these sets.

Put $Q=U\left(M_{f} \cup M_{g}\right)$ and $I=U\left(M_{f} \cap M_{g}\right)$. Using counting arguments for the vertices of $Q$ and $I$, we prove that $Q$ has exactly three corners, and that $M_{f} \cup M_{g}$ forms a minimum complex in $Q$. This, by Lemma 1 , then implies that $M_{f} \cup M_{g}$ is of degree 3.

The sets $M_{f}$ and $M_{g}$ form minimum complexes in $U\left(M_{f}\right)$ and $U\left(M_{g}\right)$, respectively (Lemma 1). Hence, a vertex is complete in $M_{f}$ if and only if it is a corner of $U\left(M_{f}\right.$ ) (same for $M_{g}$ ). Moreover, a vertex $v$ being incomplete in $M_{f}$ or $M_{g}$ is incident to a unique face $h \in M_{f} \cup M_{g}$ at a reflex angle, and if $v$ is incomplete in both $M_{f}$ and $M_{g}$ then $h \in M_{f} \cap M_{g}$ holds. We thus observe two properties:

(a) If $c$ is a corner of $Q$ or of $I$ then $c$ is a corner of at least one of $U\left(M_{f}\right)$ and $U\left(M_{g}\right)$.

(b) If $c$ is a corner of $Q$ and of $I$ then $c$ is a corner of both $U\left(M_{f}\right)$ and $U\left(M_{g}\right)$.

Now, $U\left(M_{f}\right)$ and $U\left(M_{g}\right)$ together have 6 corners, and $I$ has at least 3 corners. So, by properties (a) and (b), $Q$ has at most (and thus exactly) 3 corners.

Concerning the complex in $Q$, we recall that $M_{f}$ and $M_{g}$ already form minimum complexes. Moreover, any vertex being incomplete in $M_{f}$ or $M_{g}$ has to be incomplete in $M_{f} \cup M_{g}$ as well. So the minimum property of the complex in $Q$ is violated only if a vertex, $w$, that is complete in both sets $M_{f}$ and $M_{g}$ becomes a noncorner of $Q$. Observe that $w$ has to be a corner of both $U\left(M_{f}\right)$ and $U\left(M_{g}\right)$ then. So, from the pool of 6 corners we get from $U\left(M_{f}\right)$ and $U\left(M_{g}\right)$, already 2 are used for the noncorner $w$ of $Q$. Another 3 are used for corners of $Q$, by (a). To cover the corners of $I$ according to (a), 1 corner might be $w$, and 2 additional ones can be either yet unused corners, or corners of $Q$. In either case, by (b), 2 more corners from the pool are needed, resulting in a total of 7 corners - a contradiction. We conclude that the vertex $w$ does not exist.

Corollary 1 The set $M_{f}$ is unique for each face $f \in \mathcal{C}$. Moreover, the collection of these sets defines a partition of $\mathcal{C}$.

Proof As we have $f \in M_{f}$ for each set $M_{f}$, the uniqueness of $M_{f}$ follows from Lemma 2. To prove the partition property, let us write $f \sim g$ if $M_{f}=M_{g}$ holds for $f, g \in \mathcal{C}$. Then $\sim$ is an equivalence relation on $\mathcal{C}$. We show that $[f]_{\sim}=M_{f}$ are its equivalence classes. Clearly, $g \in[f] \sim$ implies $M_{g}=M_{f}$ and thus $g \in M_{f}$. Conversely, $g \in M_{f}$ implies $g \in M_{g} \cap M_{f}$ and thus $M_{g}=M_{f}$ by Lemma 2, that is, $g \in[f] \sim$ holds.

From Corollary 1 and Lemma 1 we know that the regions $U\left(M_{f}\right)$, for all $f \in \mathcal{C}$, partition the underlying region $U(\mathcal{C})$ of $\mathcal{C}$ into pre-triangles in a unique way. We will term the resulting pre-triangulation the $M$-skeleton of $\mathcal{C}$. Figure 9 illustrates an example. Note that not all faces of this pre-triangulation are simply connected, although there are no holes in the underlying region.

The following lemma is similar in spirit to Lemma 2. It will be needed in the design of a general flipping operation in Sect. 9. 
Lemma 3 Let $f, g \in \mathcal{C}$ be two faces that share some edge. Then either $M_{f}=M_{g}$ or the degree of $M_{f} \cup M_{g}$ is 4 .

Proof Assume $M_{f} \neq M_{g}$. Then $M_{f}$ and $M_{g}$ are disjoint, by Lemma 2. $U\left(M_{f}\right)$ and $U\left(M_{g}\right)$ have an edge $e$ in common, because $f$ and $g$ do so, and $e=U\left(M_{f}\right) \cap U\left(M_{g}\right)$ holds because we have $M_{f}=M_{g}$, otherwise. Like in the counting argument in the proof of Lemma 2 (put $I=e$ ), each endpoint $c$ of $e$ leads to a loss of at least one of the 6 possible corners of $Q=U\left(M_{f} \cup M_{g}\right)$. Thus $Q$ has at most 4 corners. Moreover, if an endpoint $c$ of $e$ is complete in $M_{f} \cup M_{g}$ but is a noncorner of $Q$, then $c$ causes a loss of two possible corners of $Q$. We conclude that $M_{f} \cup M_{g}$ is of degree 4 .

Face-reducible complexes enjoy a strong lifting property which stems from the existence of their M-skeletons, and that we are going to describe next. Define a polyhedral surface as (the graph of) a continuous and piecewise-linear function $\varphi$ whose domain is a polygonal region $\left.R \cdot \varphi\right|_{L}$ is called a facet of $\varphi$ if $L$ is a maximal interiorconnected subset of $R$ where $\varphi$ is linear.

Theorem 1 Let $\mathcal{C}$ be a face-reducible polygonal complex. Further, let $h$ be any vector assigning a height $h_{i}$ to each complete vertex $v_{i}$ of $\mathcal{C}$. There exists a unique polyhedral surface $\varphi$ for $\mathcal{C}$ and $h$, with $\varphi\left(v_{i}\right)=h_{i}$ for all $i$, and such that $\left.\varphi\right|_{f}$ is a subset of a facet of $\varphi$, for all faces $f \in \mathcal{C}$.

Proof As $\mathcal{C}$ is face-reducible, the M-skeleton of $\mathcal{C}$ exists (and is unique). Let $\mathcal{P} \mathcal{R}$ denote this pre-triangulation. The conditions required for the surface theorem in [1] to hold can be formulated as follows: For each incomplete vertex $v$ of $\mathcal{C}$, there is a unique pre-triangle $\nabla=U\left(M_{f}\right)$ of $\mathcal{P} \mathcal{R}$ such that (1) $v$ is incomplete in $M_{f}$ and (2) $v$ lies in the convex hull of the 3 corners of $\nabla$.

Consider condition (1). If $v$ is not a vertex of $\mathcal{P} \mathcal{R}$ then there is a unique $\nabla$ such that $v$ is internal to $\nabla$. Otherwise, there is a unique $\nabla$ where $v$ is a noncorner, because an internal angle larger than $\pi$ occurs in $\nabla$ at $v$. In both cases, $v$ is incomplete in the face set $M_{f}$ with $\nabla=U\left(M_{f}\right)$, by Lemma 1 . Condition (2) holds because the vertices of the convex hull of $\nabla$ are corners of $\nabla$.

\section{Planar Face Sets}

Throughout this section, let $\mathcal{C}$ be a face-reducible complex. Theorem 1 makes explicit that $\mathcal{C}$ can be lifted, in various ways, to a polyhedral surface in three-space without introducing new edges. However, not all edges of $\mathcal{C}$ might have their counterparts in this surface, for several reasons. For instance, the choice of the height vector $h$ may force more than three complete vertices to be coplanar in the lifting. Also, geometric degeneracies of $\mathcal{C}$ may be the reason; consult Fig. 2. Using our concept of M-skeleton, we are able to characterize those edges of $\mathcal{C}$ which, even under 'generic' conditions, cannot be made to show up in the surface.

Let $S$ be the set of all vertices of $\mathcal{C}$. Let $S_{\varepsilon}$ be some replacement within distance $\varepsilon$ of each vertex in $S$, for arbitrarily small $\varepsilon>0$. (We assumed $S$ to be in general position, so the order type of $S_{\varepsilon}$ equals the order type of $S$.) An $\varepsilon$-perturbation, $\mathcal{C}_{\varepsilon}$, of $\mathcal{C}$ is 
the polygonal complex with vertex set $S_{\varepsilon}$ and with the same combinatorial structure as $\mathcal{C}$. As order types are preserved, a vertex is complete in $\mathcal{C}_{\varepsilon}$ if and only if its original is complete in $\mathcal{C}$.

A subset $B$ of faces of $\mathcal{C}$ is called combinatorial planar if, for all $\varepsilon$-perturbations $\mathcal{C}_{\varepsilon}$ of $\mathcal{C}$, and for all height vectors $h$ for the complete vertices of $\mathcal{C}_{\varepsilon}$, the subset $\left.\varphi\right|_{B}$ of the surface $\varphi$ for $\mathcal{C}_{\varepsilon}$ and $h$ lies in a single plane. Recall that $\varphi$ uniquely exists by Theorem 1 .

Lemma 4 The maximal combinatorial planar face sets in a face-reducible complex $\mathcal{C}$ are the pre-triangles $U\left(M_{f}\right), f \in \mathcal{C}$, of its $M$-skeleton.

Proof Consider such a pre-triangle $U\left(M_{f}\right)$. By definition of an M-skeleton, $M_{f}$ is of degree 3 . So exactly 3 vertices are complete in $M_{f}$. For every height vector $h$ for $\mathcal{C}$, the plane through the corresponding 3 points in space determines a possible surface for $M_{f}$, because all other vertices of the complex formed by $M_{f}$ are incomplete in $M_{f}$, and thus are incomplete in $\mathcal{C}$. By the uniqueness of this surface, all surface vertices for $M_{f}$ have to lie in this plane. This reasoning remains true for any $\varepsilon$-perturbation $\mathcal{C}_{\varepsilon}$ of $\mathcal{C}$. Therefore $M_{f}$ is combinatorial planar.

It remains to show that $M_{f}$ is maximal under the condition of being combinatorial planar. Consider the linear system that, given $\mathcal{C}$ and $h$, describes the unique surface according to Theorem 1; see [1]. The variables in this system are the heights for all the vertices of $\mathcal{C}$, including the incomplete ones. Let now $B$ be any proper superset of $M_{f}$. By the structure of the system, the heights of the vertices in $B$ only depend on heights of vertices for supersets $B^{\prime}$ of $B$. Moreover, the heights for any such $B^{\prime}$ can be described by using only the heights for the vertices that are complete in $B^{\prime}$ (Theorem 1 applied to $B^{\prime}$ ). On the other hand, the face set $M_{f} \subset B$, by definition, is maximal under the condition of having 3 complete vertices. That is, every superset $B^{\prime} \supseteq B$ has degree at least 4 . Therefore, when using the equations of the system, the vertex heights for $B$ cannot be described by only 3 of the system variables (unless the system is overdetermined because of geometric artifacts; these can be removed by $\varepsilon$-perturbing both $\mathcal{C}$ and $h$ ). Moreover, no other system with the same solution space can describe the heights for $B$ with fewer variables, because our system takes into account all the vertex heights. But a plane in three-space is determined by 3 parameters, and we conclude that there exist $\varepsilon$-perturbations $\mathcal{C}_{\varepsilon}$ of $\mathcal{C}$ and $h_{\varepsilon}$ of $h$ such that the subset $\left.\varphi\right|_{B}$ of the surface $\varphi$ for $\mathcal{C}_{\varepsilon}$ and $h_{\varepsilon}$ is not coplanar. That is, $B$ is not combinatorial planar.

\section{Combinatorial Projectivity}

We now define a notion of projectivity (i.e., liftability) for polygonal complexes which is robust with respect to variations in lifting height and, at the same time, outrules complexes that are projective (or non-projective) only because of geometric artifacts.

Consider an arbitrary polygonal complex $\mathcal{C}$. Define an exact lift of $\mathcal{C}$ as a polygonal surface whose set of edges projects exactly to the set of edges of $\mathcal{C}$. In an exact lift of $\mathcal{C}$, no edge of $\mathcal{C}$ is allowed to flatten out, and no face of $\mathcal{C}$ is allowed to fold at 
new edges. Not every polygonal complex admits an exact lift; perturbed Schlegel diagrams (Fig. 1) are an example.

We are interested in complexes whose space of exact lifts has maximal dimension. It will turn out that this dimension is bounded from above by the degree, $k$, of $\mathcal{C}$. Let us call the complex $\mathcal{C}$ combinatorial projective if, for a random ${ }^{3} \varepsilon$-perturbation $\mathcal{C}_{r}$ of $\mathcal{C}$, the space of exact lifts of $\mathcal{C}_{r}$ has dimension $k$ with probability 1 . Intuitively speaking, for almost all perturbations of a combinatorial projective complex, almost all height vectors for its $k$ complete vertices will lead to an exact lift.

Our aim is to characterize the class of combinatorial projective complexes. The next assertion formulates a main observation in this context. Its proof is immediate from the proof of Lemma 4.

Corollary 2 Let $\mathcal{C}$ be a face-reducible complex, and let $h_{r} \in[0,1]^{k}$ be a random height vector for the $k$ complete vertices of $\mathcal{C}_{r}$. Then, with probability 1 , the edges in the surface for $\mathcal{C}_{r}$ and $h_{r}$ bijectively correspond to the edges of the $M$-skeleton of $\mathcal{C}$.

Theorem 2 A polygonal complex $\mathcal{C}$ is combinatorial projective if and only if the $M$-skeleton of $\mathcal{C}$ exists and is identical to $\mathcal{C}$.

Proof For the class of face-reducible complexes, the assertion is true by Corollary 2. To complete the proof, we assume that $\mathcal{C}$ is not face-reducible (i.e., the M-skeleton of $\mathcal{C}$ does not exist) and argue that $\mathcal{C}$ then cannot be combinatorial projective.

Faces of $\mathcal{C}$ not being part of some set of degree $3(\mathcal{C}$ is supposed to contain at least one such face) have more than 3 corners. So, for each such face $f$, some internal edge can be added to split $f$ into two faces, without changing any vertex from incomplete to complete. We keep adding such edges until a face-reducible complex $\mathcal{C}^{\prime}$ is obtained. Let $B(f)$ denote the set of faces of $\mathcal{C}^{\prime}$ that a face $f$ of $\mathcal{C}$ splits into. Now, $\mathcal{C}$ has to have some face $f$ whose set $B(f)$ is not contained in a single pre-triangle of the M-skeleton of $\mathcal{C}^{\prime}$, as $\mathcal{C}$ would be face-reducible, otherwise. Therefore, in the surface $\varphi^{\prime}$ for $\mathcal{C}_{r}^{\prime}$ and $h_{r}$, the set $\left.\varphi^{\prime}\right|_{B(f)}$ is not part of a single facet of $\varphi^{\prime}$ with probability 1 ; see Corollary 2. By the uniqueness of the surface theorem, there is no other way of constructing the required surface for $\mathcal{C}_{r}$ and $h_{r}$. This implies that, with probability 1 , the space of exact lifts for $\mathcal{C}_{r}$ is strictly less in dimension than the degree of $\mathcal{C}_{r}$. That is to say, $\mathcal{C}$ is not combinatorial projective.

\section{Corollary 3 Every combinatorial projective complex is a pre-triangulation.}

The proof of Theorem 2 also shows the following: Every polygonal complex $\mathcal{C}$ can be refined to a face-reducible one without changing its degree $k$. Therefore, the space $\mathcal{L}$ of 'face-embedding' lifts of $\mathcal{C}$, in the sense of Theorem 1 , is of dimension at most $k$, and this bound is achieved if $\mathcal{C}$ is face-reducible. The space $\mathcal{E}$ of exact lifts of $\mathcal{C}$ is the complement of a finite union of linear subspaces in $\mathcal{L}$, each subspace representing the lifts that flatten out a fixed edge of $\mathcal{C}$. Thus $\mathcal{E}$ is either empty or the dimension of $\mathcal{E}$ is $k$ as well.

\footnotetext{
${ }^{3}$ Drawn uniformly for each vertex from its surrounding $\varepsilon$-disk.
} 
If a complex $\mathcal{C}$ is not face-reducible then-apart from special cases caused by geometric degeneracies - for almost all choices of heights for $\mathcal{C}$ 's complete vertices, certain faces of $\mathcal{C}$ will split, in every surface which respects these heights. We thus have:

Corollary 4 Theorem 1 cannot be extended beyond the class offace-reducible polygonal complexes.

Figure 7 shows an example for a face-reducible complex which is not combinatorial projective; the complex is not identical to its M-skeleton (see Fig. 9). A pseudotriangulation which is not combinatorial projective is shown in Fig. 11(a) (upper drawing); the four pseudo-triangles form a face set which is combinatorial planar. However, combinatorial planarity of a set of two or more faces does not necessarily destroy combinatorial projectivity. For example, the pre-triangle in Fig. 8(d) constitutes both a combinatorial projective complex and a combinatorial planar face set of size three. Note also that, although each combinatorial projective complex is a pretriangulation, not all of its faces need to be pre-triangles; see the pentagonal star in Fig. 8(c).

\section{Combinatorial Regularity}

In this section we focus on convex projection surfaces. We introduce the classes of combinatorial regular complexes and face-honest complexes and elaborate on their interrelation. Face-honest complexes are of interest for two more reasons: Loosely speaking, each such complex can be lifted to a surface so that its faces live in pairwise different planes. Moreover, these complexes allow for easy modification with flipping operations; see Sect. 12.

Let $\varphi$ be some polyhedral surface. An edge $e$ of $\varphi$ is called convex if there exists a line segment $\ell$ that nowhere lies above $\varphi$ and that intersects $e$ at exactly one point interior to both $e$ and $\ell$. Edges of $\varphi$ which are not convex are called reflex. Note that the image $\left.\varphi\right|_{e^{\prime}}$ of each boundary edge $e^{\prime}$ of the domain of $\varphi$ is a convex edge.

A polygonal complex $\mathcal{C}$ is called combinatorial regular if $\mathcal{C}$ is combinatorial projective and there exists some $\varepsilon$-perturbation of $\mathcal{C}$ that admits an exact lift where all edges are convex. By Corollary 3, combinatorial regular complexes are pre-triangulations. Known examples are (constrained) Delaunay triangulations [18], and more generally, the constrained regular pseudo-triangulations [1]. Schlegel diagrams [13] and thus Voronoi diagrams are not combinatorial regular (though they are well known to be regular in the classical sense) because these complexes are not combinatorial projective, apart from the special case of triangulations.

Lemma 5 Let $\mathcal{C}$ be a combinatorial regular complex. Then each internal vertex of $\mathcal{C}$ is complete.

Proof Let $\mathcal{C}_{\varepsilon}$ and $h$ define an exact lift that witnesses the combinatorial regularity of $\mathcal{C}$. Consider an incomplete vertex $v$ of $\mathcal{C}$, and let $f$ be the unique face of $\mathcal{C}$ where $v$ is a noncorner. To get a contradiction, suppose $v$ is an internal vertex of $\mathcal{C}$. As there 
is an internal angle greater than $\pi$ in $f$ at $v$, there exists some line segment $\ell \subset U(\mathcal{C})$ such that $\ell$ crosses all the edges of $\mathcal{C}$ incident to $v$. But $\mathcal{C}$ is combinatorial regular, so the corresponding edges in the surface $\varphi$ for $\mathcal{C}_{\varepsilon}$ and $h$ are all convex. Therefore $\varphi(f)$ cannot be part of a single facet of $\varphi-$ a contradiction to the definition of $\varphi$.

A (face-reducible) polygonal complex is termed face-honest if $M_{f}=\{f\}$ holds for each of its faces $f$. Being identical to its M-skeleton, every face-honest complex $\mathcal{C}$ is a pre-triangulation that is combinatorial projective, by Theorem 2. Moreover, all pre-triangles of $\mathcal{C}$ are single faces. Figure 9 gives an illustration. On the other hand, even when a pseudo-triangulation is combinatorial regular, it is not necessarily facehonest. Figure 8(d) reveals this fact. Triangulations are always face-honest: All their vertices are complete, so their individual triangles constitute the only possible face sets of degree 3. See Fig. 3 for the interaction of face-honest complexes and combinatorial regular complexes with other classes.

Below we derive some results for the case where the underlying region $U(\mathcal{C})$ of the complex $\mathcal{C}$ in question is a polygon.

Lemma 6 Let $\mathcal{C}$ be a face-reducible complex, and suppose each internal vertex of $\mathcal{C}$ is complete. If $U(\mathcal{C})$ is a polygon then $\mathcal{C}$ is a face-honest pseudo-triangulation.

Proof Let $f$ be a face of $\mathcal{C}$. As $\mathcal{C}$ is face-reducible, the set $M_{f}$ exists. Each vertex $v$ that is incomplete in $M_{f}$ is also incomplete in $\mathcal{C}$, and thus $v$ is a vertex of $U(\mathcal{C})$, by assumption. Therefore, if $U(\mathcal{C})$ is required to be a polygon, then the pre-triangle $U\left(M_{f}\right)$ is just a pseudo-triangle, and $M_{f}$ contains a single face, $U\left(M_{f}\right)=f$.

Lemmas 5 and 6 combine to the following statement.

Theorem 3 Let $\mathcal{C}$ be a combinatorial regular complex whose underlying region is a polygon. Then $\mathcal{C}$ is a face-honest pseudo-triangulation.

Corollary 5 Let $\mathcal{P} \mathcal{T}$ be a pseudo-triangulation without internal vertices in a polygon $R$. Then $\mathcal{P} \mathcal{T}$ is face-honest.

Proof As $\mathcal{P} \mathcal{T}$ contains no internal vertices, each internal edge of $\mathcal{P} \mathcal{T}$ is a diagonal of $R$. This implies that $\mathcal{P} \mathcal{T}$ is combinatorial regular. The assertion now follows from Theorem 3.

\section{Locally Convex Surfaces}

We show next that combinatorial regular complexes - and thus certain pre-triangulations-arise from graphs of locally convex functions on polygonal domains. This generalizes results in [1] where locally convex functions are introduced and utilized in the context of pseudo-triangulations. The relationship between locally convex functions and pseudo-simplicial complexes in higher dimensions is discussed in detail in $[5]$. 
Let a polygonal domain $R$ be given. A surface $\varphi$ on $R$ is called locally convex if each edge of $\varphi$ is convex. ${ }^{4}$ Let now $S \subset R$ be a finite set of points that includes all the vertices of $R$. Further, let $h$ be a vector that assigns an upper height bound $h(v)$ to each point $v \in S$. We define $F_{h}$ as the maximal (i.e., highest) locally convex surface on $R$ that satisfies $F_{h}(v) \leq h(v)$ for all $v \in S$.

The surface $F_{h}$ exists and is unique: Any surface $\varphi$ that consists of a single facet, $\left.\varphi\right|_{R}$, is locally convex and can be lowered to satisfy $h$, and $F_{h}$ is the pointwise maximum of all possible surfaces on $R$ that are locally convex and satisfy $h$. The facets of $F_{h}$ project to the faces of a polygonal complex in $R$ which we denote by $\mathcal{C}\left(F_{h}\right)$.

The next lemma follows from results in [5]. We give a more direct proof here.

Lemma 7 All vertices of $\mathcal{C}\left(F_{h}\right)$ are included in $S$. For each complete vertex $v$ of $\mathcal{C}\left(F_{h}\right), F_{h}(v)=h(v)$ holds.

Proof Let $w$ be a vertex of $\mathcal{C}\left(F_{h}\right)$. Assume $w \notin S$. Then $w$ has to be complete in $\mathcal{C}\left(F_{h}\right)$ : If $w$ lies on the boundary of $R$ then clearly no face of $\mathcal{C}\left(F_{h}\right)$ has an internal angle larger than $\pi$ at $w$. If $w$ is an internal vertex of $\mathcal{C}\left(F_{h}\right)$ then the same is true, because the image $F_{h}(e)$ of each edge $e$ incident to $w$ is a convex edge. But for any complete vertex $v$ of $\mathcal{C}\left(F_{h}\right)$ we must have $v \in S$ and $F_{h}(v)=h(v)$, because otherwise the height of $v$ can be increased without violating the local convexity of the surface or the restrictions in $h$, in contradiction to the maximality of $F_{h}$.

Let us define a generic situation (for height vector $h$ and point set $S$ ) as one where, for some $\varepsilon>0$, the maximal locally convex surface for any $\varepsilon$-perturbation $h_{\varepsilon}$ of $h$ and $S_{\varepsilon}$ of $S$ has the same combinatorial structure as the surface $F_{h}$.

If the underlying polygonal region $R$ is a convex polygon, then $F_{h}$ is the lower convex hull [21] of the spatial point set $\left\{\left(\begin{array}{c}v \\ h(v)\end{array}\right) \mid v \in S\right\}$. If, in addition, the situation is generic then $\mathcal{C}\left(F_{h}\right)$ is a regular triangulation [10, 17]. This fact generalizes for arbitrary polygonal regions $R$ as follows.

Theorem 4 Under generic conditions, the complex $\mathcal{C}\left(F_{h}\right)$ is a pre-triangulation that is combinatorial regular.

Proof By Lemma 7, $F_{h}(v)=h(v)$ holds for each complete vertex $v$ of $\mathcal{C}\left(F_{h}\right)$. Together with the genericity assumption on $h$, this implies that the space of exact lifts of $\mathcal{C}\left(F_{h}\right)$ has dimension (at least) its degree. Since the underlying point set $S$ is generic, too, it follows that $\mathcal{C}\left(F_{h}\right)$ is combinatorial projective. But $h$ is a height vector for $\mathcal{C}\left(F_{h}\right)$ such that all edges of $F_{h}$ are convex. We conclude that $\mathcal{C}\left(F_{h}\right)$ is combinatorial regular. Moreover, $\mathcal{C}\left(F_{h}\right)$ is a pre-triangulation, by Corollary 3 .

Corollary 6 If the domain $R$ of $F_{h}$ is a polygon, then $\mathcal{C}\left(F_{h}\right)$ is a face-honest pseudotriangulation in the generic case.

Proof Combine Theorem 3 and Theorem 4.

\footnotetext{
${ }^{4}$ Equivalently, $\varphi(\ell)$ defines a convex function, for every line segment $\ell \subset R$ that does not cross the boundary of $R$. This definition of locally convexity is used in $[1,5]$. 


\section{A General Flipping Scheme}

Flip operations are a common tool for locally modifying polygonal complexes. A general class where flip operations have been defined are the pseudo-triangulations. The repertoire includes Lawson flips [16], exchanging flips [20, 24], and removing flips and their inverses [1, 19]. Still, several interesting classes of complexes, in particular the combinatorial regular complexes whose relevance as optimal surfaces is documented in Sect. 8, cannot be reached and modified with these flip operations. The combinatorial regular complex in Fig. 8(c) serves as an example.

Let $\mathcal{C}$ be a face-reducible complex. Below we define a flip operation that applies to any internal edge $e$ of $\mathcal{C}$, in a way such that face-reducibility is retained in the resulting complex. The flip operation is based on locally convex surfaces and on maximal face sets of degree 3 . All the flips known for triangulations and pseudo-triangulations arise as special cases of this operation.

OpERATION FLIP $(e)$. Let $f$ and $g$ be the two faces of $\mathcal{C}$ incident to $e$. Consider the subcomplex $\mathcal{C}_{e}=M_{f} \cup M_{g}$ of $\mathcal{C}$. Choose a height for each complete vertex of $\mathcal{C}_{e}$ such that, if $M_{f} \neq M_{g}$, the edge $\left.\varphi\right|_{e}$ is reflex in the surface $\varphi$ for $\mathcal{C}_{e}$. Let the vector $h$ contain these heights and, in addition, the entry $\infty$ for each incomplete vertex of $\mathcal{C}_{e}$. Replace $\mathcal{C}_{e}$ by $\mathcal{C}\left(F_{h}\right)$.

$\operatorname{FLIP}(e)$ deletes a subset $E$ of edges of $\mathcal{C}$ and creates a subset $E^{\prime}$ of edges disjoint from $E$. Note that $E \neq \emptyset$ because $\operatorname{FLIP}(e)$ always deletes the edge $e$. That is, each internal edge of $\mathcal{C}$ is flippable with this operation. We might have $E^{\prime}=\varnothing$ if the flip is removing. The cardinalities of $E$ and $E^{\prime}$ may be large, though. We offer a detailed discussion of the flip types covered by the operation FLIP in Sect. 10.

Lemma 8 FLIP $(e)$ constructs a unique and combinatorial regular subcomplex $\mathcal{C}\left(F_{h}\right)$.

Proof If $M_{f}=M_{g}$ then $M_{f} \cup M_{g}$ is of degree 3. For any entries in $h$ for these 3 complete vertices, we obviously have $\mathcal{C}\left(F_{h}\right)$ unchanged. If $M_{f} \neq M_{g}$ then $M_{f} \cup M_{g}$ is of degree 4, by Lemma 3. The corresponding 4 entries in $h$ were chosen such that $e$ yields a reflex edge in the surface for $M_{f} \cup M_{g}$. This implies that the respective 4 surface vertices are not coplanar. So $\mathcal{C}\left(F_{h}\right)$ remains unchanged when $h$ is $\varepsilon$-perturbed. Moreover, even a large change of $h$ does not alter $\mathcal{C}\left(F_{h}\right)$ as long as the surface image of $e$ is reflex, because the (three-dimensional) order type of the 4 surface vertices stays the same. This shows that $\operatorname{FLIP}(e)$ constructs a unique surface. In particular, $h$ is generic. Moreover, an $\varepsilon$-perturbation of the vertices in $M_{f} \cup M_{g}$ will not change the surface combinatorially. Thus generic conditions are given, and $\mathcal{C}\left(F_{h}\right)$ is combinatorial regular, by Theorem 4 .

Theorem 5 The class of face-reducible complexes is closed under the operation FLIP. Pre-triangulations are the smallest class having this property, among the classes listed in Fig. 3.

Proof Let $\mathcal{C}$ be some face-reducible complex in the polygonal region $R=U(\mathcal{C})$. Let $e$ be an internal edge of $\mathcal{C}$, and denote with $\mathcal{C}^{\prime}$ the complex that is obtained from $\mathcal{C}$ by applying $\operatorname{FLIP}(e)$. 
The subcomplex $\mathcal{C}\left(F_{h}\right)$ of $\mathcal{C}^{\prime}$ constructed by $\operatorname{FLIP}(e)$ is combinatorial regular, by Lemma 8. Thus $\mathcal{C}\left(F_{h}\right)$ is a pre-triangulation in $Q=U\left(M_{f} \cup M_{g}\right)$, for $f$ and $g$ being the faces incident to $e$. In the complement $R \backslash Q$, the complex $\mathcal{C}^{\prime}$ coincides with $\mathcal{C}$. Moreover, by nature of $\operatorname{FLIP}(e)$, the M-skeletons of $\mathcal{C}^{\prime}$ and $\mathcal{C}$ are identical in $R \backslash Q$. Hence, the part of $\mathcal{C}^{\prime}$ in $R \backslash Q$ is a face-reducible complex.

If, in addition, $\mathcal{C}$ is a pre-triangulation then there exists a partition of $\mathcal{C}$ into face sets $B_{i}$ such that $U\left(B_{i}\right)$ is a pre-triangle and no edge of $\mathcal{C}$ is internal to $U\left(B_{i}\right)$, for each set $B_{i}$. So $B_{i}$ is of degree 3, which implies $B_{i} \subseteq M_{b}$ if $b \in B_{i}$ holds for a face $b$ external to $Q$. This implies that $B_{i}$ is not affected by $\operatorname{FLIP}(e)$ in this case. Consequently, the part of $\mathcal{C}^{\prime}$ in $R \backslash Q$ is a pre-triangulation.

But the concatenation of two pre-triangulations (or of two face-reducible complexes) is a complex of the same type, as is easily seen from the definition of such complexes.

To complete the proof, we observe that there exist Delaunay triangulations constrained by $R$ that can be flipped to a pre-triangulation which is neither combinatorial projective nor a pseudo-triangulation. Figure 11(b) exemplifies this fact. In view of the containment relations in Fig. 3, this shows that any class closed under FLIP has to include the class of pre-triangulations.

Note that Theorem 5 does not imply that all face-reducible complexes (or pretriangulations) within a given region $R$ can be reached by the operation FLIP, if one starts with a fixed one.

\section{Instances of FLIP}

Let us discuss the effect of the operation FLIP in different scenarios. First of all, the interested reader may convince himself that FLIP is able to simulate the exchanging flips in Fig. 4 and the removing flips in Fig. 5; see Sect. 1.2. In the figures for the present section (Figs. 10, 11, and 12) the edge $e$ to be flipped is shown in bold, and only the region $U\left(M_{f} \cup M_{g}\right)$ that gets restructured is displayed. Here $f$ and $g$ are the two faces incident to $e$. Completeness of vertices (indicated by black dots) is with respect to the set $M_{f} \cup M_{g}$, and is not necessarily the same in the entire complex. Edges created by the respective flip are drawn as dashed lines. By Lemma 3, the degree of the face set $M_{f} \cup M_{g}$ is at most 4. Therefore, the region $U\left(M_{f} \cup M_{g}\right)$ (let us call it $Q$ in the discussion below) has either 4 or 3 corners.

The case of 4 corners for $Q$ leads to a generalization of the exchanging flip for pre-triangles; see Fig. 10. We have $M_{f} \neq M_{g}$ and the degree of $M_{f} \cup M_{g}$ is 4 . As in a usual exchanging flip, the completeness status of all vertices is preserved (apart from possible vertices internal to $Q$ that are necessarily incomplete and are removed by the flip along with their incident edges). More than one edge may be created, as is shown in flip (a) as well as in flip (b). Consequently, FLIP is not a symmetric operation, in general: Flipping one edge (or all the edges in any order) that just have been created need not give the initial complex.

The case of 3 corners for $Q$ constitutes a generalization of the removing flip; see Fig. 11(a). All edges and (incomplete) vertices internal to $Q$ get removed. No new edges are created and a single pre-triangle, $Q$, remains. The degree of $M_{f} \cup M_{g}$ is 
Fig. 10 Two exchanging flips

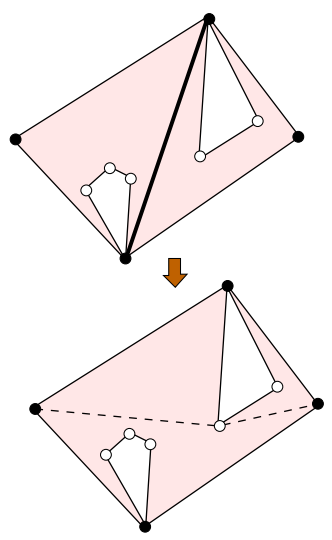

Fig. 11 (a) Removing flip. (b) Many flips

Fig. 12 (a) Correct flip. (b) 'Wrong' flip (a)

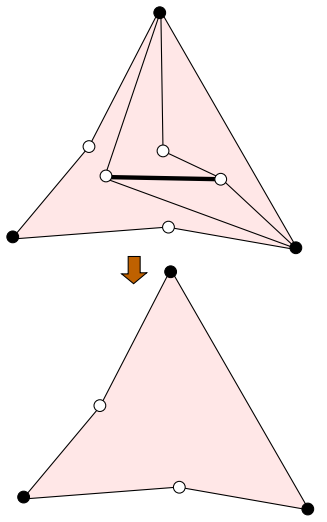

(a)
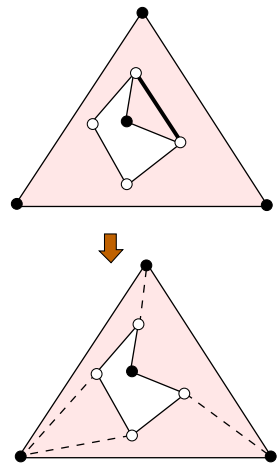

(b)
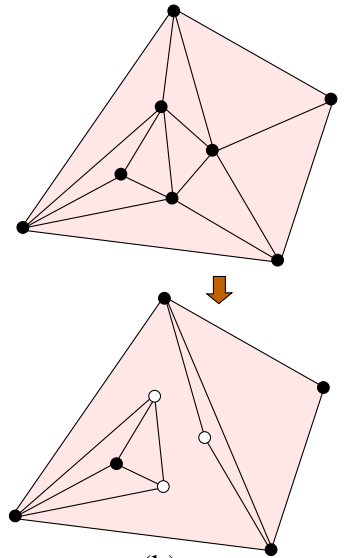

(b)

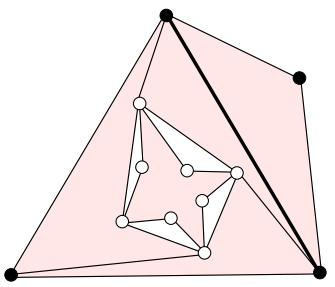

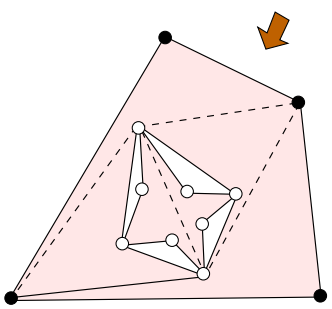

(a)

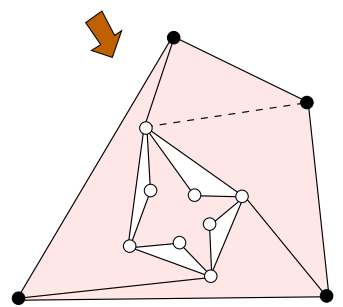

(b) 
Fig. 13 Delaunay minimum complex

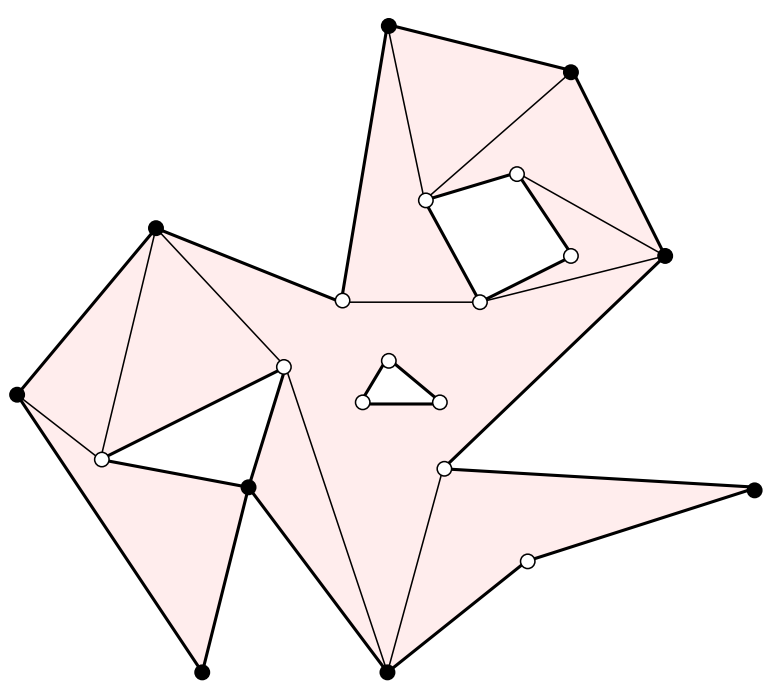

either 3 as in our example and in Fig. 5 (left), or is 4 as in Fig. 4 (right). This depends on whether $M_{f}=M_{g}$ or not. In the negative case, a noncorner of $Q$ which is an endpoint of the flipped edge $e$ changes its status from complete to incomplete.

A natural question is why $\operatorname{FLIP}(e)$ is based on maximal face sets of degree 3 rather than on single faces of $\mathcal{C}$. Figure 12 provides an answer. The flip (a) is in accordance with the definition of FLIP and retains face-reducibility. In flip (b) that is solely based on the two faces incident to the flipped edge, face-reducibility is lost, and with it, the applicability of the surface theorem (Theorem 1) and its advantages, which will be exploited next.

\section{Delaunay Minimum Complex}

By the well-known paraboloid lifting function $h(x)=|x|^{2}$, Delaunay triangulations $[2,11]$ in a convex region $R$ correspond to maximal locally convex surfaces generated by the vertex heights $h(x)$. (In this easy case, such surfaces are just lower convex hulls of the lifted vertices.) This correspondence is extended to nonconvex polygons $R$ in [1] and leads to Delaunay triangulations constrained by (the edges of) $R$. We define below a unique complex of smallest combinatorial size in an arbitrary polygonal region $R$ that still shows the desired 'Delaunay properties': an analog of local Delaunayhood, and the reachability by improving flip operations.

Let $p$ be the height vector for the vertices of $R$ such that $p(c)=|c|^{2}$ for each corner $c$ of $R$, and $p(v)=\infty$ for each noncorner $v$ of $R$. Consider the complex $\mathcal{D}_{R}=\mathcal{C}\left(F_{p}\right)$. We will assume generic conditions for $R$ henceforth, in the sense that $\varepsilon$-perturbations of its vertices (and the changes of vertex heights caused by them) leave the maximal locally convex surfaces above combinatorially unchanged. Then the height vector $p$ is generic, too, and by Theorem 4 the complex $\mathcal{D}_{R}$ is a pretriangulation that is combinatorial regular. Moreover, $\mathcal{D}_{R}$ is a minimum complex in $R$ because, by Lemma 7, its only vertices are those of $R$ and each noncorner of $R$ 
is incomplete in $\mathcal{D}_{R}$. We term $\mathcal{D}_{R}$ the Delaunay minimum complex of $R$. Figure 13 gives an example.

Observe that $\mathcal{D}_{R}$ differs from the constrained Delaunay triangulation [18] of $R$, unless $R$ is a convex polygon and $\mathcal{D}_{R}$ is the (classical) Delaunay triangulation of the vertices of $R$. In case $R$ is a polygon, $\mathcal{D}_{R}$ is a minimum pseudo-triangulation which is face-honest, by Corollary 6. The latter structure has been also considered in [22] and is a constrained regular pseudo-triangulation as defined in [1].

The class of all possible face-reducible complexes in a given region $R$ is connected under the operation FLIP in the following sense.

Theorem 6 Let a polygonal region $R$ be given. Each face-reducible complex in $R$ can be transformed to the Delaunay minimum complex $\mathcal{D}_{R}$ by using finitely many operations FLIP.

Proof Let $\mathcal{C}$ be a face-reducible complex in $R$. We construct a finite sequence of flips that transforms $\mathcal{C}$ into $\mathcal{D}_{R}$. Let $h$ be a height vector for the vertices of $\mathcal{C}$, with $h(c)=|c|^{2}$ for each corner $c$ of $R$, and with heights for the remaining vertices of $\mathcal{C}$ being sufficiently large such that $\mathcal{C}\left(F_{h}\right)=\mathcal{D}_{R}$.

Apply $h$ to the complete vertices of $\mathcal{C}$. This defines a unique surface $\varphi$ on $R$ by Theorem 1. If $\varphi$ contains reflex edges then choose such an edge $\left.\varphi\right|_{e}$, transform $\mathcal{C}$ to a new complex $\mathcal{C}^{\prime}$ by performing $\operatorname{FLIP}(e)$ for the edge $e$ of $\mathcal{C}$, and put $\mathcal{C}=\mathcal{C}^{\prime}$ and repeat.

The complexes $\mathcal{C}_{i}$ created above are face-reducible, by Theorem 5. So the procedure is well-defined. We first prove that the created surfaces $\varphi_{i}$ are pairwise different. ${ }^{5}$ Let $\operatorname{FLIP}(e)$ transform $\mathcal{C}_{i}$ to $\mathcal{C}_{i+1}$. Denote by $Q$ the region restructured by $\operatorname{FLIP}(e)$, and let $\mathcal{S}$ and $\mathcal{S}^{\prime}$, respectively, be the subcomplex of $\mathcal{C}_{i}$ and $\mathcal{C}_{i+1}$ in $Q$. By adding all internal edges of $\mathcal{S}^{\prime}$ to $\mathcal{C}_{i}$ (and splitting faces of $\mathcal{C}_{i}$ accordingly) we obtain a polygonal complex $A$ in $R$. Extend $h$ to a height vector $h_{A}$ for the complete vertices of $A$ such that the surface for $A$ and $h_{A}$ is $\varphi_{i}$. (This is possible although $A$ is not face-reducible, in general.) Similarly, extend $h$ to $h_{A}^{\prime}$ such that the surface for $A$ and $h_{A}^{\prime}$ is $\varphi_{i+1}$. Then $h_{A}^{\prime} \leq h_{A}$ holds (element-wise, with strict inequality for some elements), because $e$ is the only internal edge for $\mathcal{S}$ and $\left.\varphi_{i}\right|_{e}$ is reflex, and all internal edges $e^{\prime}$ of $\mathcal{S}^{\prime}$ have a convex image $\left.\varphi_{i+1}\right|_{e^{\prime}}$. But surface heights can be shown to strictly decrease with the height vector (see [1], Lemma 5.2). This implies that the surfaces $\varphi_{i}$ are pairwise different.

In fact, the complexes $\mathcal{C}_{i}$ are pairwise different as well: If $\mathcal{C}_{i}$ and $\mathcal{C}_{j}$ have different sets of complete vertices then clearly $\mathcal{C}_{i} \neq \mathcal{C}_{j}$. Otherwise, by the uniqueness of $\varphi_{i}$ for fixed $h, \varphi_{i} \neq \varphi_{j}$ implies $\mathcal{C}_{i} \neq \mathcal{C}_{j}$. Observe next that no new vertices are created by the operation FLIP. But the number of polygonal complexes with vertices from a fixed set of $n$ points is finite (in fact, exponential in $n$ ). We conclude that the procedure above terminates with a surface $\varphi^{*}$ where all edges are convex.

In the corresponding complex $\mathcal{C}^{*}$, the only vertices that are complete are the corners of $R$ : Any other complete vertex $v$ of $\mathcal{C}^{*}$ would be incident to edges whose

\footnotetext{
${ }^{5}$ Thanks go to Paco Santos for pointing out, in the context of pseudo-triangulations, the following elegant argument.
} 
images are reflex in $\varphi^{*}$, by the choice of $h(v)$. This implies $\varphi^{*}=F_{h}$ by the uniqueness of $F_{h}$. Observe that $\mathcal{C}\left(F_{h}\right)=\mathcal{D}_{R}$ is the M-skeleton of $\mathcal{C}^{*}$. If $\mathcal{C}^{*} \neq \mathcal{D}_{R}$ then $\mathcal{C}^{*}$ contains internal edges (and possibly, internal and incomplete vertices) that have no counterparts in $F_{h}$. As long as such an edge $e$ exists, we apply $\operatorname{FLIP}(e)$. For the two faces $f$ and $g$ incident to $e$, we have $M_{f}=M_{g}$. Therefore, $\operatorname{FLIP}(e)$ removes all edges internal to $U\left(M_{f}\right)$ (in particular, the edge $e$ ) and creates no edges. The number of such flips is bounded by the number of edges of $\mathcal{C}^{*}$. The surface $\varphi^{*}$ remains unchanged, and the complex $\mathcal{D}_{R}$ is the result.

Theorem 6 is rather general. Flips can be applied in any order to edges with reflex images, and we even can drop the requirement that such edges have to be flipped before edges without surface counterparts. Moreover, when starting with a pre-triangulation this class is never left; see Theorem 5. We remark that Theorem 6 is a generalization of the optimality theorem for pseudo-triangulations in [1].

As a special case, let $R$ be a polygon, and let $\mathcal{P} \mathcal{T}$ be some minimum pseudotriangulation in $R$ without internal vertices. Then $\mathcal{P} \mathcal{T}$ is face-honest by Corollary 5. Let $e$ be an edge of $\mathcal{P} \mathcal{T}$ which is incident to two pseudo-triangles $\nabla$ and $\nabla^{\prime}$. We have $M_{\nabla}=\nabla \neq \nabla^{\prime}=M_{\nabla^{\prime}}$ and the region affected by $\operatorname{FLIP}(e)$ is $Q=\nabla \cup \nabla^{\prime}$. Moreover, $Q$ has exactly 4 corners by Lemma 3. So $\operatorname{FLIP}(e)$ is an exchanging flip; see Sect. 10. Let $h$ be the height vector used above to define $D_{R}=\mathcal{C}\left(F_{h}\right)$. We may call $\operatorname{FLIP}(e)$ a Delaunay flip if the edge $\left.\varphi\right|_{e}$ in the surface $\varphi$ for $\mathcal{P} \mathcal{T}$ and $h$ is reflex. As $\operatorname{FLIP}(e)$ transforms $\mathcal{P} \mathcal{T}$ to a minimum pseudo-triangulation of $R$, and $D_{R}$ is a complex of this type, we obtain:

Corollary 7 Any minimum pseudo-triangulation of a polygon $R$ can be flipped to $D_{R}$ by repeated application of Delaunay flips and without leaving the class of minimum pseudo-triangulations.

If Delaunay flips are performed in a well-chosen order then $O\left(n^{2}\right)$ flips are sufficient if $R$ has $n$ vertices, as can be seen by adapting a result in [1]. Note finally that the edges of $D_{R}$ do not form a subset of the edges of the constrained Delaunay triangulation [18] of $R$, in general.

\section{Face-Honest Flipping}

Throughout this section, we restrict attention to polygonal complexes whose underlying region $R$ is a polygon. We show that any triangulation $\mathcal{T}$ in $R$ can be flipped to the complex $\mathcal{C}\left(F_{h}\right)$, for any given (generic) height vector $h$ for the vertices of $\mathcal{T}$, in a way such that each intermediate complex is a pseudo-triangulation. (We allow $\mathcal{T}$ to contain internal vertices.) In fact, all obtained complexes are face-honest and thus are combinatorial projective, except in cases where an internal vertex has to be removed in the subsequent flip and face-honesty is impossible to keep. This guarantees that only standard flips for pseudo-triangulations are performed. We start with a technical lemma. 
Lemma 9 Let $\mathcal{P} \mathcal{T}$ be a pseudo-triangulation in $R$, and let $v$ be an internal and complete vertex of $\mathcal{P} \mathcal{T}$ with at least 4 incident edges. Then at least one such edge can be flipped by exchange such that no edge incident to $v$ is created.

Proof Let $Q$ be the union of the faces of $\mathcal{P} \mathcal{T}$ incident to $v$, and let $\mathcal{C}$ be the pseudotriangulation in $Q$ defined by these faces. For an edge $v x$ of $\mathcal{C}$, define $\alpha(v x)$ as the internal angle at $v$ of the union of the two faces of $\mathcal{C}$ adjacent in $v x$. Imagine that removing edge flips are applied to all edges $v u$ where $u$ is a non-corner of $Q$ that is complete in $\mathcal{C}$. Each such flip makes a unique vertex incomplete. This vertex is different from $v$ (and thus is $u$ ) because $\alpha(v u)<\pi$ holds by our assumption on $u$. So $v$ stays complete in the obtained complex, and therefore at least 3 edges of $\mathcal{C}$ are still incident to $v$. Such an edge $v w$ can be flipped in $\mathcal{C}$ by exchange, and without creating an edge incident to $v$, if $\alpha(v w)<\pi$. But this is guaranteed for at least one such edge, because we assumed $v$ to be incident to at least 4 edges of $\mathcal{C}$.

Theorem 7 Let $R$ be a polygon and let $\mathcal{T}$ be a triangulation in $R$. Moreover, let $h$ be a height vector for the vertices of $\mathcal{T}$, and assume generic conditions for the complex $\mathcal{C}\left(F_{h}\right)$. Then $\mathcal{T}$ can be transformed by FLIP to $\mathcal{C}\left(F_{h}\right)$, in a way such that all intermediate complexes are face-honest pseudo-triangulations, except immediately before a vertex is removed.

Proof Denote with $\mathcal{P} \mathcal{T}$ the current complex obtained from flipping; initially, $\mathcal{P} \mathcal{T}=\mathcal{T}$. Recall that all vertices of $\mathcal{T}$ are complete. We construct a desired sequence of flips that terminates with $\mathcal{P} \mathcal{T}=\mathcal{C}\left(F_{h}\right)$. Let $\varphi$ denote the unique surface that results from $\mathcal{P} \mathcal{T}$ when $h$ is applied to all complete vertices. We say that an internal vertex $v$ of $\mathcal{P} \mathcal{T}$ fulfills the hull condition if $\left.\varphi\right|_{v}$ lies strictly below the lower convex hull of its neighbored vertices in $\varphi$.

Step (1): As long as there exists an internal vertex $v$ of $\mathcal{P} \mathcal{T}$ that violates the hull condition, we do the following. Apply exchanging flips to the edges incident to $v$ until only 3 such edges remain. This is possible by Lemma 9. After each flip all the internal vertices of $\mathcal{P} \mathcal{T}$ are complete, because this was true before the flip, and the flip was exchanging. Two more applications of FLIP to $v$ 's remaining edges first make $v$ incomplete (and $\mathcal{P} \mathcal{T}$ temporary non-projective and hence non face-honest), and then remove $v$, leaving all internal vertices complete again.

Step (2): While there exists an edge $e$ in $\mathcal{P} \mathcal{T}$ such that $\left.\varphi\right|_{e}$ is reflex, do the following: Apply $\operatorname{FLIP}(e)$. If $e$ is exchanged then no vertex alters its completeness status. If $e$ gets removed then an endpoint of $e$ which is a vertex of $R$ becomes incomplete: Each internal vertex fulfills the hull condition before the flip, and $\left.\varphi\right|_{e}$ was reflex. So all internal vertices stay complete after $\operatorname{FLIP}(e)$. If some internal vertices now violate the hull condition then repeat from Step (1). (Note that $\mathcal{P} \mathcal{T}$ need not be a triangulation any more.)

The total number of flips performed in Step (1) is clearly $O\left(n^{2}\right)$, if $\mathcal{T}$ contains $n$ vertices. In Step (2) only edges with a reflex image are flipped. The number of these flips is finite by the arguments in the proof of Theorem 6 . In each created complex all internal vertices are complete, except immediately before a vertex is removed. So, with these exceptions, each such complex is a face-honest pseudo-triangulation, by Lemma 6. 
In particular the final complex, $\mathcal{P} \mathcal{T}^{*}$, is face-honest and therefore combinatorial projective. The surface $\varphi^{*}$ for $\mathcal{P} \mathcal{T}^{*}$ contains no reflex edge. This implies that, for each edge $e$ of $\mathcal{P} \mathcal{T}^{*}$, its image $\left.\varphi^{*}\right|_{e}$ is a convex edge. As only vertices that violate the hull condition are removed, and such vertices cannot belong to $\mathcal{C}\left(F_{h}\right)$, we conclude $\mathcal{P} \mathcal{T}^{*}=C\left(F_{h}\right)$.

We conjecture that only quadratically many flips are performed in Step (2). This would yield a flip sequence of total length $O\left(n^{2}\right)$. Theorem 7 implies that the complex $\mathcal{C}\left(F_{h}\right)$ for polygons is a pseudo-triangulation. A non-algorithmic proof for this fact has been given in Sect. 8 (Corollary 6).

A result related to Theorem 7 has been proved in [14]. Namely, any triangulation $\mathcal{T}$ can be flipped to the complex $\mathcal{C}\left(F_{h}\right)$ such that no edges with convex images in the corresponding surface are flipped, and such that all intermediate complexes are combinatorial projective (with the above exceptions before vertex removal).

\section{Concluding Remarks}

We have introduced the concept of pre-triangulations, a relaxation of triangulations that goes beyond the frequently used concept of pseudo-triangulations. Less intuitive at first sight, pre-triangulations turned out to be more natural than pseudo-triangulations in questions concerning liftability and flippability of polygonal complexes-even in the most simple case where the underlying region is a convex polygon.

One of the central tools used in our developments is the completeness status of the vertices of a polygonal complex. (In)completeness of a vertex depends on the (non)convexity of its incident internal angles and thus on the order type of the vertices of the complex in the end. This enabled us to characterize complexes which exhibit the 'robust' lifting property we called combinatorial projectivity.

We did not address algorithmic issues in this paper. Deciding combinatorial projectivity of a given polygonal complex in an efficient way is one of them. In view of Theorem 2 this question reduces to identifying the M-skeleton of the complex or detecting its non-existence. As a simple approach we may try to constructafter $\varepsilon$-perturbing the vertices - a surface for the complex in question, using random heights for its complete vertices. The surface construction mainly means resolving a system of $n$ linear equations [1] where $n$ is the number of vertices. If the system has a solution then the edges of the resulting unique surface correspond to the M-skeleton with probability one (see Corollary 2). Otherwise, the M-skeleton does not exist.

Other relevant algorithmic questions are finding short flip sequences within particular complex classes, and implementing the operation FLIP efficiently. These questions are discussed in detail in a separate paper [4]. Emphasis is laid on constructing locally convex functions, and Delaunay-type complexes in particular.

Acknowledgements The authors would like to thank an anonymous referee for his various constructive and clarifying comments. 


\section{References}

1. Aichholzer, O., Aurenhammer, F., Krasser, H., Brass, P.: Pseudo-triangulations from surfaces and a novel type of edge flip. SIAM J. Comput. 32, 1621-1653 (2003)

2. Aurenhammer, F.: Voronoi diagrams-a survey of a fundamental geometric data structure. ACM Comput. Surv. 23, 345-405 (1991)

3. Aurenhammer, F.: A criterion for the affine equivalence of cell complexes in $R^{d}$ and convex polyhedra in $R^{d+1}$. Discrete Comput. Geom. 2, 49-64 (1987)

4. Aurenhammer, F., Hackl, T., Krasser, H.: Short flip sequences for constructing the Delaunay triangulation. Manuscript (2006)

5. Aurenhammer, F., Krasser, H.: Pseudo-simplicial complexes from maximal locally convex functions. Discrete Comput. Geom. 35, 201-221 (2006)

6. Bern, M., Eppstein, D.: Mesh generation and optimal triangulation. In: Du, D.-Z., Hwang, F. (eds.) Computing in Euclidean Geometry. Lecture Notes Series on Computing, vol. 4, pp. 47-123. World Scientific, Singapore (1995)

7. Brøndsted, A.: An Introduction to Convex Polytopes. Springer, Berlin (1983)

8. Crapo, H., Whiteley, W.: Plane self stresses and projected polyhedra. Struct. Topol. 20, 55-78 (1993)

9. Edelsbrunner, H.: Algorithms in Combinatorial Geometry. Springer, Berlin (1987)

10. Edelsbrunner, H., Shah, N.R.: Incremental topological flipping works for regular triangulations. Algorithmica 15, 223-241 (1996)

11. Fortune, S.: Voronoi diagrams and Delaunay triangulations. In: Du, D.-Z., Hwang, F. (eds.) Computing in Euclidean Geometry. Lecture Notes Series on Computing, vol. 4, pp. 225-265. World Scientific, Singapore (1995)

12. Goodman, J.E., Pollack, R.: Multidimensional sorting. SIAM J. Comput. 12, 484-507 (1983)

13. Grünbaum, B.: Convex Polytopes. Wiley-Interscience, London (1967)

14. Hackl, T.: Manipulation of pseudo-triangular surfaces. Master thesis, Institute for Theoretical Computer Science, University of Technology, Graz, Austria (2004)

15. Hurtado, F., Noy, M., Urrutia, J.: Flipping edges in triangulations. Discrete Comput. Geom. 22, 333346 (1999)

16. Lawson, C.L.: Properties of $n$-dimensional triangulations. Comput. Aided Geom. Des. 3, 231-246 (1986)

17. Lee, C.W.: Subdivisions and triangulations of polytopes. In: Goodman, J.E., O'Rourke, J. (eds.) Handbook of Discrete and Computational Geometry, 2nd edn., pp. 383-406. CRC Press, Boca Raton (2004)

18. Lee, D.T., Lin, A.K.: Generalized Delaunay triangulation for planar graphs. Discrete Comput. Geom. 1, 201-217 (1986)

19. Orden, D., Santos, F.: The polytope of non-crossing graphs on a planar point set. Discrete Comput. Geom. 33, 275-305 (2005)

20. Pocchiola, M., Vegter, G.: Topologically sweeping visibility complexes via pseudo-triangulations. Discrete Comput. Geom. 16, 419-453 (1996)

21. Preparata, F.P., Shamos, M.I.: Computational Geometry: An Introduction. Springer, New York (1985)

22. Rote, G., Schulz, A.: A pointed Delaunay pseudo-triangulation of a simple polygon. In: Proceedings of the 21st European Workshop on Computational Geometry, pp. 77-80 (2005)

23. Steinitz, E.: Polyeder und Raumeinteilungen. Enzyklopaedie der Math. Wiss. III AB 12, Leipzig (1916)

24. Streinu, I.: A combinatorial approach to planar non-colliding robot arm motion planning. In: Proceedings of the 41st IEEE Symposium on FOCS, pp. 443-453 (2000) 